$\Phi(\rho z)$ model and Monte Carlo model. He also addresses these general considerations in his own contribution to the present issue, with a special emphasis on the role of computer development (see Microsc. Microanal. Microstruct. 3 (1992)).

The following chapters of the book cover very thoroughly the different methods available for quantitative EPMA analysis. They are written by all the experts who have gradually contributed to the general improvement of our knowledge in this domain over the past decades : V. Scott and G. Love, J.D. Brown, R. Packwood, R. Myklebust and D.E. Newbury, P. Karduck and W. Rehbach. I wish however to underline the important contribution of two groups of french scientists, who have been at some time the students of Raimond Castaing. Jean Louis Pouchou and Françoise Pichoir quite consistently describe the PAP procedure which relies on the fundamental principles of microanalysis and intends to provide a realistic description of $\Phi(\rho z)$. They include in an appendix the complete set of 826 entries in the data basis which they used to test their procedure! On the other hand, Jean Hénoc and Françoise Maurice advocate for the Monte Carlo procedure. All involved steps are described with full details and the listing of the program that they have elaborated for simulating X-ray depth distribution is reported as an appendix. These two examples demonstrate, if necessary, the vitality of the french school of microanalysis founded by Raymond Castaing.

The final two chapters of the book introduce subjects which are presently getting increased recognition as demonstrated with other recent publications discussed further on. The first one (written by Dale Newbury and collaborators) deals in fact with an old topic : quantitative compositional mapping. It goes back to the early days of microanalysis but has really burst out with the advent of modern, efficient and low-cost computers. The second one (introduced by two leading experts in the field, Dave Williams and Joe Goldstein) is devoted to X-ray microanalysis on thin foils. It has now become an essential component of the analytical transmission electron microscope.

In conclusion, this is a very well documented reference book for anybody involved in the practical use of X-ray microanalysis on bulk specimens, which is clearly demonstrated through the application described in some of its chapters.

\title{
Transmission electron energy loss spectrometry in materials science edited by M.M. DISKO, C.C. AHN and B. FULTZ (Minerals, Metals and Materials Society, TMS, 1992)
}

"Electron energy loss spectrometry (EELS) is a relatively new addition to the group of diffraction, imaging and spectroscopic techniques available for the study of materials with the transmission electron microscope". This statement by the editors in their preface for this quite recent publication of 270 pages on EELS methods and applications, should however be modulated. Actually the elemental information carried in electron energy loss spectra has been identified nearly fifty years ago by Hillier and Baker, following the first studies on inelastic scattering of electrons from solids by Rudberg in 1936. However the message seems to have been neglected for several decades, during which period the analysis of $\mathrm{X}$ ray photons emitted under the action of the incident electron probe, has established itself as the most powerful method for microanalysis under the leading role of $\mathbf{R}$. Castaing.

In fact, the influence of Castaing and his coworkers L. Henry and A. El Hili, has also been prominent for the reemergence of electron energy loss spectroscopy as a tool for local chemical characterization. The introduction, in 1962, of the Castaing and Henry energy filter as a combination of a magnetic prism and an electrostatic mirror, in the electron microscope column, 
has largely contributed to the development of this technique. Its present impact is well demonstrated in the contributions of Reimer et al. and of Krivanek et al. to the present Microsc. Microanal. Microstruct. issue.

Nevertheless it is obvious that EELS has established itself as an essential component of the analytical electron microscope only during the seventies, independantly in France and in USA with the thesis studies of Colliex and Isaacson, then followed by Egerton, Leapman and many others. Progress in all aspects (instrumentation, scattering theory, quantitative analysis of spectra and applications) has been very thoroughly discussed by R. Egerton in his textbook "Electron Energy Loss Spectroscopy in the Electron Microscope" (Plenum Press, NY 1986) which has remained, together with the EELS Atlas due to O. Krivanek and C. Ahn (1983), the reference document for the whole generation of scientists coming to this technique during the last few years.

The new monograph edited by Disko, Ahn and Fultz contains ten chapters derived from the oral presentations at a symposium "Applications of Transmission EELS in materials science" which was held during the 120th annual TMS meeting at New Orleans in january 1991. Its scope is to respond to the growing demand from materials scientists to combine this new analytical tool and problem-solving approaches, in order to achieve productive EELS experiments. To meet these requirements, the five first chapters are dedicated to energy loss methods that are general to all areas of materials studies. They cover experimental techniques and instrumentation ( $R$. Egerton), quantitative analysis (R. Leapman), spatially resolved EELS (C. Colliex), EELS fine structures (P. Rez). In all cases, the availability of efficient PEELS devices has contributed to great changes in data acquisition, processing and interpretation. It is particularly true for the methods of multiple least squares fitting on difference spectra discussed by Leapman, which push the detectability limits to useful values in the $10^{-4}$ atomic concentration range. When combined with a small probe and a high flux of primary electrons delivered from a field emission source, the identification of single individual atoms is then accessible, see Colliex. Finally the high energy resolution now available, has made EELS very competitive with $\mathrm{X}$ ray absorption spectroscopy studies on a synchrotron line to record and investigate fine structures (of XANES or EXAFS types) on core edges, more specially when they concern inhomogeneous specimens (see P. Rez). Simultaneously, theoretical advances (molecular orbitals calculations, band structure and multiple scattering descriptions, many particle effects...) have also largely contributed to increase the practical interest of using these fine structures, as beautifully demonstrated by $\mathrm{R}$. Brydson $e t$ al.

In fact one originality of this book is to devote its second half to specific areas of materials science : minerals (R. Brydson et al.), ceramics and catalysts (J. Bentley), metals (J. Okamoto et al.), semiconductors (P. Batson) and advanced materials (N. Zaluzec), which all bear witness of the vitality and richness of EELS as a local analytical tool in the electron microscope.

Great care has been taken by the editors to harmonize the different chapters so that the whole volume is readily self understandable, the texts are clearly written with many illustrative figures, many unpublished data are included. Altogether the volume elegantly fulfills its scope and can therefore be recommended to a large population of readers.

\section{Microscopy. The key research tool. Compositional imaging. New microscopies} A special publication of the Electron Microscopy Society of America, 1992

Finally I feel that it is worthwile to mention briefly at the end of this Books Review section this special EMSA publication for its 50th anniversary. It is the first time that this society has published an entire volume of full-length research papers in a journal format. It is to be put to the credit of 